\section{FUENTES DE CONOCIMIENTO EN LOS PROCESOS DE INNOVACIÓN EMPRESARIAL: LAS SPIN-OFF UNIVERSITARIAS EN ANDALUCÍA 1}

\author{
Oihana Valmaseda \\ Instituto de Estudios Sociales Avanzados (IESA), CSIC
} ovalmaseda@iesa.csic.es

\author{
Nuria Hernández \\ Instituto Universitario de Ciencias Políticas y Sociales (IUCPS) \\ Universidad de la Laguna \\ nherher@ull.es
}

\begin{abstract}
This paper explores the knowledge adquisition strategy developed by university spin-offs, in the context of the theoretical discussion on the presence of various knowledge sources used by companies for their technology and innovation strategies. The empirical basis of this study is a survey to the population of spin-offs in the Andalusian university system. The findings confirm that the companies studied combine in-house $R \& D$ and acquired knowledge from their relations with external agents. Literature on innovation shows a lack of empirical analysis using spin-off companies as a unit of analysis. Therefore, the results of this paper have not only theoretical relevance but also offer important implications in the field of innovation policy.
\end{abstract}

KEY WORDS: University spin-off; knowledge sources; innovation strategy.

\section{INTRODUCCIÓN}

En las últimas décadas se han producido importantes cambios en lo que respecta al proceso de creación de conocimiento que, a su vez, han modificado tanto el papel desempeñado por las universidades, destacadas productoras de conocimiento, como sus relaciones con los agentes del entorno (Gibbons et al., 1994; Etzkowitz y Leydesdorff, 1997). Existen evidencias crecientes de los beneficios que la transferencia de conocimiento desde la universidad reporta al crecimiento económico de las regiones. Pero además, también existe un considerable interés por parte
KNOWLEDGE SOURCES IN FIRM INNOVATION PROCESSES: THE CASE OF UNIVERSITY SPIN-OFFS IN ANDALUSIA

RESUMEN: El presente trabajo explora la estrategia de adquisición de conocimiento de las spin-off universitarias, en el marco de la discusión teórica sobre la concurrencia de distintas fuentes de conocimiento en la estrategia tecnológica e innovadora de las empresas. La base empírica de esta investigación la constituye una encuesta a la población de empresas creadas en el sistema universitario andaluz. La investigación permite observar que, en sus procesos de innovación, las empresas estudiadas combinan el conocimiento que se deriva de la realización de I+D interna con el adquirido a través de su relación con agentes externos. En la literatura sobre innovación, existe cierta carencia de estudios empíricos que usen como unidad de análisis a las empresas spin-off. Por ello, los resultados de este trabajo ofrecen interés para este campo científico y también importantes implicaciones en el ámbito de las políticas públicas de innovación.

PALABRAS CLAVE: Spin-off universitaria; fuentes de conocimiento; estrategia de innovación.

de los decisores en el ámbito público y entre los administradores de la universidad en comprender las implicaciones políticas y de gestión de este nuevo paradigma.

La creación de empresas basadas en conocimiento generado en la universidad, las denominadas spin-off universitarias, son un importante mecanismo de transferencia de conocimiento vinculado a la comercialización de resultados de actividades de I+D. Salvo por la particularidad del origen del conocimiento que da lugar a su constitución y diversos aspectos en el orden estructural y organizacional que caracterizan estas organizaciones, se trata de empre- 
sas que, al igual que otras, para sostener su competitividad en contextos cada vez más cambiantes e inciertos, se implican en procesos de innovación cuya creciente complejidad requiere la utilización de diferentes formas y fuentes de conocimiento.

A pesar de esta coincidencia, para el caso de las empresas que no surgen en el ámbito académico la literatura muestra el interés y dedicación de investigadores en la comprensión y explicación de las diferentes estrategias de innovación. No obstante, para el caso de las spin-off universitarias se trata de un terreno insuficientemente explorado. Existen evidencias de que las nuevas empresas innovadoras, o start-ups, son muy activas en el uso de la investigación pública en sus proyectos de investigación (Cohen et al., 2002), pero, en general, la literatura adolece de planteamientos conceptuales y empíricos que focalicen su atención en las fuentes de conocimiento a las que acceden las empresas académicas en sus procesos de innovación.

El proceso de innovación empresarial ha sido ampliamente examinado desde diferentes perspectivas. Entre éstas, cabe destacar la que lo explica a través del modelo interactivo, que señala la gran complejidad del proceso innovador y la necesidad de fuentes de conocimiento diversas que tienen como origen tanto a la propia empresa como a numerosos agentes externos. Este hecho lleva a las empresas a establecer una red de interacciones con diversos actores de su entorno. Así, han sido objeto de investigación los posibles agentes que representan una fuente de conocimiento para las empresas, las razones por las cuales una organización empresarial decide acceder a conocimiento externo, los mecanismos a través de los cuales las empresas obtienen dicho conocimiento y los beneficios derivados de dicha relación.

Si bien es cierto que la adquisición de conocimiento externo se presenta como una opción necesaria para llevar a cabo los procesos de innovación, no siempre resulta ser suficiente. Por ello, es necesario enfatizar el papel que desempeña la realización de actividades de I+D interna y el carácter estratégico de las mismas. La realización de estas actividades está relacionada con la capacidad de absorción (Cohen y Levinthal, 1989, 1990; Lane et al., 2006), que hace referencia al conjunto de capacidades internas de las organizaciones que permiten tanto la generación de ideas innovadoras y el aprendizaje intraorganizacional, como explotar y asimilar el conocimiento de otros agentes externos. Así, las empresas realizan otras actividades complejas relacionadas con la vigilancia tecnológica y de mercado, la formación y el diseño, que permiten reconocer y valorar el conocimiento existente o disponible, adaptarlo a los objetivos e integrarlo en las funciones de la empresa para ponerlo en valor y obtener en lo posible mejores resultados (Bender y Laestadius, 2005). Estas ideas se plasman en trabajos como los que plantean escenarios de complementariedad entre la realización de actividades de I+D interna y la interacción con agentes externos, y que son entendidos como estrategias compatibles en la búsqueda de conocimiento. En consecuencia, es recomendable estudiar este fenómeno observando la adopción conjunta y relacionada de estas estrategias de innovación (Mowery, 1983; Cassiman y Veugelers, 2006; Romijn et al., 2002; Freel, 2005 y Caloghirou et al., 2004).

En este trabajo nos preguntamos si, para el caso de las spin-off universitarias, estas estrategias concurren o no y cuáles son los factores que conducen a la empresa a optar entre la adquisición de conocimiento de fuentes externas, centrarse exclusivamente en sus capacidades internas o bien combinar en diferentes proporciones ambas fuentes de conocimiento. Es relevante conocer cuál es la opción escogida por este tipo de empresas en lo que tiene que ver con la decisión de búsqueda y utilización de las diversas fuentes de conocimiento estratégicas para su actividad, tratándose de organizaciones que surgen como resultado de un proceso de transferencia de conocimiento desde la universidad.

En este sentido, el objetivo de esta investigación es aportar una fundamentación teórica y empírica que permita avanzar en el estudio de las fuentes de conocimiento para la innovación en las empresas de tipo spin-off, adoptando los avances teóricos y metodológicos generados en estudios cuya unidad de análisis son empresas que no surgen del entorno académico. Para tal fin, en primer término, se describirá el fenómeno de la creación de empresas en el contexto académico. En segundo término, se estudiarán las diferentes fuentes de conocimiento a las que acceden las spin-off en Andalucia para sus procesos de innovación, examinando la influencia que ejercen en dichos procesos la investigación pública realizada por universidades y organismos públicos de investigación, así como por empresas del sector privado. El trabajo se organiza en las siguientes 
secciones a partir de la presente introducción: en el apartado segundo efectuamos una revisión de la literatura como fundamento teórico del trabajo. En el apartado tercero se expone la metodología empleada en la investigación. En el apartado cuarto avanzamos los resultados obtenidos con respecto a las preguntas formuladas. Finalmente, presentaremos las conclusiones que ofrecen implicaciones en el ámbito de las políticas públicas de innovación.

\section{Antecedentes}

\subsection{Revisión del concepto, tipologías y determinantes de la competitividad de las spin-off universitarias}

Si bien la literatura relativa a la creación de empresas en el contexto universitario está aumentando, existe una evidente ausencia de claridad en lo que respecta a la definición del concepto "spin-off universitaria" (Pirnay, 1998). Una importante corriente de investigación en este ámbito trata de identificar los criterios de clasificación y comprensión de este complejo fenómeno. Muchos de estos autores han prestado atención a la necesidad de definir una tipología de spin-offs universitarias (Smirlor et al., 1990; Carayannis et al., 1998; Bellini et al., 1999; Rappert et al., 1999; Clarysse et al., 2000; Klofsten et al., 2000; Steffensen et al., 2000). Sus trabajos revelan dos principales dimensiones para la clasificación: por un lado, el estatus de los individuos involucrados en los procesos de creación de las nuevas empresas, de otro, la naturaleza del conocimiento transferido desde la universidad a la nueva empresa (Pirnay et al., 2003).

Otra de las conclusiones que se extraen de la revisión de la literatura es que existen diferentes tipos de empresas basadas en conocimiento universitario cuyo desarrollo se ve afectado por diversos factores. Por tanto, no se puede hablar del "emprendizaje" académico como un fenómeno que se comporta de manera homogénea en cualquier contexto (Mustar, 2000; Mustar et al., 2006). La existencia de estas ambigüedades en el uso del concepto spin-off universitaria, así como la ausencia de consideración de los factores específicos que caracterizan a los sistemas universitarios y de innovación en los que se enmarcan estas empresas, dificultan sobremanera los avances en esta línea de investigación.
El interés de clarificar e integrar las definiciones existentes y de categorizar los tipos de spin-off reside, por una parte, en la posibilidad de atribuir características particulares a cada uno de los tipos de empresa identificados y, por otra, en la observación de distintas actitudes y comportamiento con respecto a las estrategias de adquisición de conocimiento relevante. Así, tal y como recoge Pirnay et al. (2003), dependiendo de la naturaleza del conocimiento transferido y del estatus de las personas implicadas en la creación de la spin-off, es posible detectar características distintas en lo que respecta a la oportunidad de negocio, al emprendedor y a los recursos y capacidades de las empresas.

Otras corrientes de investigación estudian los mecanismos y factores que explican el éxito de estas nuevas empresas (Mustar et al., 2006). Los estudios empíricos pueden clasificarse en tres tradiciones teóricas diferentes. Una la conforman un grupo de autores que centran su atención en el estudio de la relación que las spin-off mantienen con su organización de origen. Es la conocida como perspectiva institucional, interesada en el análisis de la influencia de la organización de origen en la configuración inicial de recursos de la spin-off y en su posterior desarrollo (Carayannis et al., 1998; Steffensen et al., 2000; Meyer, 2003; Moray y Clarysse, 2005; Clarysse et al., 2005).

La segunda, denominada del modelo de negocio, tiene origen en la literatura de dirección y gestión empresarial (Chesbrough y Rosenbloom, 2002; Zott y Amit, 2005), y centran sus análisis en el sector de actividad, la tecnología y las diferentes combinaciones producto-mercado adoptadas por las spin-offs (Bower, 2003; Druilhe y Garnsey, 2004; Stankiewicz, 1994; Chiesa y Piccaluga, 2000; Mustar, 2002).

Por último, la visión basada en los recursos de la organización empresarial (Barney et al., 2001; Shane y Stuart, 2002; Hellmann y Puri, 2000; Bower, 2003; Heirman y Clarysse, 2004; Druilhe y Garnsey, 2004), cuyos trabajos se centran en aquellos recursos de la empresa que la diferencian de sus competidores y que, además, representan una fuente de ventaja competitiva. Entre estos recursos, identificamos como aquellos que pueden explicar el comportamiento de las empresas de nueva creación (Barney, 1991; Brush et al., 2001) los siguientes: los financieros (Heirman y Clarysse, 2004; Mustar, 1997; Wright et al., 2004), los tecnológicos

ARBOR Vol. 188753 enero-febrero [2012] 211-228 ISSN: 0210-1963 
(Autio, 1997; Autio y Lumme, 1998; Hindle y Yencken, 2004; Heirman y Clarysse, 2004), los humanos (Fontes, 2001; Pirnay et al., 2003; Shane y Stuart, 2002; Sholten et al., 2001) y los sociales (Lindelhof y Losten, 2004; Autio, 1997; Nicolaou y Birley, 2003).

Teniendo en cuenta la revisión de investigaciones en este campo, la definición adoptada en la presente investigación es la que considera como spin-off a las empresas creadas en el ámbito académico (universidades o centros públicos de investigación) para la explotación comercial de los resultados de la investigación u otros fines. La diversidad implícita en esta definición tiene implicaciones que justifican el análisis desde un punto de vista empírico de las fuentes de conocimiento utilizadas por estas empresas en el contexto regional escogido.

\subsection{Estrategias de adquisición de conocimiento para la innovación empresarial}

Las explicaciones del proceso innovador en las empresas han experimentado una importante evolución. La perspectiva neoclásica, bajo lo que se conoce como enfoque lineal, propició un tratamiento del conocimiento científico como algo exógeno al proceso innovador y establecía que su influencia en dicho proceso tenía lugar de manera secuencial o lineal. Así, un descubrimiento científico daría lugar a un desarrollo tecnológico que, a su vez, permitiría obtener un nuevo producto para finalmente producir un beneficio económico (Kline y Rosenberg, 1986). Este planteamiento no reconocía la retroalimentación en el proceso, ni el aprendizaje que se genera en el mismo. Sin embargo, gracias a las contribuciones del enfoque evolucionista, sabemos que existe otra forma de explicar el proceso de innovación empresarial a través de lo que se conoce como modelo interactivo.

Dentro de la teoría económica evolucionista, la perspectiva de la empresa basada en los recursos constituye una base teórica sólida para la explicación de las razones que llevan a las empresas a colaborar con otros agentes y obtener, como fruto de esa relación, los recursos y capacidades complementarias que le permitan sostener su ventaja competitiva. Este enfoque reconoce como estrategias válidas para la innovación la compra de tecnología e $I+D$, o bien la cooperación con agentes externos, pero sin olvidar las competencias internas de la empresa en varios ámbitos: el organizacional, el de mercado y el tecnológico, que son la clave para el máximo aprovechamiento de los recursos externos (Tidd, 2000). Una correcta gestión y equilibrio entre capacidades internas y externas suponen la clave competitiva (Teece et al., 1997), lo que posibilita la realización de una variedad de actividades que van desde las vinculadas a la explotación a aquellas más encaminadas a la exploración, que componen lo que la literatura coincide en denominar como estrategias de búsqueda, dirigidas a la resolución de problemas en las empresas (March, 1991).

Una capacidad interna fundamental es la de absorción (Cohen y Levinthal, 1989), indispensable para que la empresa identifique y valore convenientemente el conocimiento que obtiene de fuentes externas (Grimpe y Sofka, 2009). Por tanto, la capacidad de absorción es determinante en el proceso de adquisición de conocimiento externo. Pero a su vez, el acceso, la utilización y la aplicación de conocimiento externo, junto al propio proceso de interacción de la empresa con una universidad $u$ organismo gubernamental de investigación, contribuye a la mejora y al aprendizaje interno. Por ello, cabe esperar que, en lugar de decidir si hacer uso de capacidades internas o bien acceder a recursos y capacidades externos complementarios, puede resultar más acertado contemplar la complementariedad de ambas estrategias (Mowery, 1983; Cassiman y Veugelers, 2006; Romijn et al., 2002; Freel 2003; Freel 2005; Caloghirou et al., 2004).

En esta línea, Cassiman y Veugelers (2006) confirman, para el caso de Bélgica y el sector manufacturero, la existencia de complementariedad entre las diferentes actividades para la innovación y, en consecuencia, el beneficio mutuo derivado de la coexistencia de ambas. El trabajo concluye que aquellas empresas que desarrollan una de las estrategias, esto es, el desarrollo de actividades de I+D interna o bien la adquisición externa de $I+D$, ven reducida su capacidad de introducir nuevos productos o mejorados en comparación con aquellas empresas en las que concurren ambas estrategias. Asimismo, los resultados que arroja la investigación de Caloghirou et al. (2004), a partir de una encuesta realizada a 558 empresas innovadoras de países como Alemania, Dinamarca, Francia, Grecia, Holanda, Italia y Reino Unido, confirman las tesis de que la combinación de las capacidades internas de I+D y la alta cualificación del personal, con la interacción y el acceso a recursos de conocimiento externos, además de reforzar la capacidad de 
absorción de la empresa, permite generar valor añadido y aumentar su capacidad innovadora.

No obstante, otros trabajos no concluyen de igual manera $y$, por tanto, no es posible hablar de consenso en lo que respecta a la idoneidad de combinar ambas estrategias. Así, hallazgos empíricos, como los del trabajo de Laursen y Salter (2006), apuntan efectos de sustitución entre el refuerzo de las capacidades de I+D internas y el acceso a fuentes externas de conocimiento para la innovación. Existe relación entre ambas estrategias pero se trata de una interacción de signo negativo que los autores explican aduciendo cierta resistencia por parte del personal de las empresas al percibir una excesiva atención hacia los recursos que se obtienen de agentes externos.

Actualmente, los avances y esfuerzos se encaminan a identificar los determinantes estructurales y organizativos que influyen en las estrategias de búsqueda de conocimiento y patrones de innovación, entre los que destacan el tamaño empresarial, el sector de actividad, la intensidad tecnológica y la inversión en $\mathrm{I}+\mathrm{D}$, que permiten explicar con mayor rigor y concreción las estrategias adoptadas por el sector empresarial. Los estudios se han centrado frecuentemente en el análisis de los patrones de innovación en empresas pequeñas (Romijn, 2002; Freel, 2005) o bien en empresas con distinto nivel de intensidad tecnológica (Santamaría et al., 2009; Grimpe et al., 2009). La consideración del tamaño empresarial ha generado de manera reiterada hipótesis con conclusiones contrapuestas. Laursen y Salter (2006) concluyen que el tamaño de la empresa es significativo para el caso de las innovaciones incrementales, pero no para las radicales. Otros trabajos profundizan en la eficiencia con la que afrontan las empresas las relaciones con agentes externos. Así, hay resultados que indican que las empresas de menor tamaño tienen ventaja sobre las grandes en la explotación de conocimiento externo (Acs et al., 1994) y, además, obtienen mayor rendimiento. Igualmente, se observa una mayor propensión a establecer vínculos, por ejemplo con universidades, en el caso de las empresas de mayor tamaño (Link y Rees, 1990).

Otra variable clásica que puede predecir la estrategia de búsqueda y adquisición de conocimiento es el sector de actividad atendiendo a su intensidad tecnológica $(O C D E$, 1997), esto es, si la empresa es de baja, media o alta tecno- logía. Gran parte de los trabajos incluyen en sus muestras a empresas de sectores de alta tecnología, como robótica (Katila, 2002; Katila y Ahuja 2002) o biotecnología (Shan et al., 1994), y se constata la relación positiva entre las estrategias de búsqueda de recursos externos y la innovación. Sin embargo, otras investigaciones han tenido como fin la identificación de distintos patrones de comportamiento entre sectores de distinta intensidad tecnológica. En este sentido, caben señalar investigaciones recientes como la de Santamaría et al. (2009), quienes para el caso español confirman que las empresas españolas de baja y media intensidad tecnológica, más allá de la realización de actividades de $I+D$, utilizan en el marco de su estrategia de innovación otras actividades como los procesos de diseño, la contratación de consultoría y la formación. Grimpe y Sofka (2009) también confirman patrones de búsqueda de conocimiento que difieren atendiendo a la intensidad tecnológica del sector. La investigación que realiza con una muestra de 4.500 empresas de 13 paises europeos le permite concluir que las empresas de baja y media intensidad tecnológica buscan conocimiento en clientes y competidores fundamentalmente $y_{\text {, }}$ in embargo, las universidades y los proveedores tecnológicos son la fuente principal de conocimiento en empresas de sectores de alta intensidad tecnológica.

Considerando como base, por un lado, los planteamientos empíricos que estudian los patrones de búsqueda de conocimiento de las empresas y la complementariedad entre fuentes internas y externas de conocimiento $y$, por otro, aquellos que profundizan en los factores que condicionan la elección de una determinada estrategia de adquisición de conocimiento, la presente investigación pretende describir las fuentes de conocimiento utilizadas por las spin-off en los procesos de innovación, así como estudiar la existencia de distintos comportamientos en función del tamaño de las empresas, el área de conocimiento, el sector de actividad y la actividad exportadora de la empresa.

\section{Metodología}

\section{1. Ámbito de estudio y recogida de datos}

La población objeto de estudio en la investigación ${ }^{2}$, está constituida por el conjunto de empresas spin-off creadas

ARBOR Vol. 188753 enero-febrero [2012] 211-228 ISSN: 0210-1963

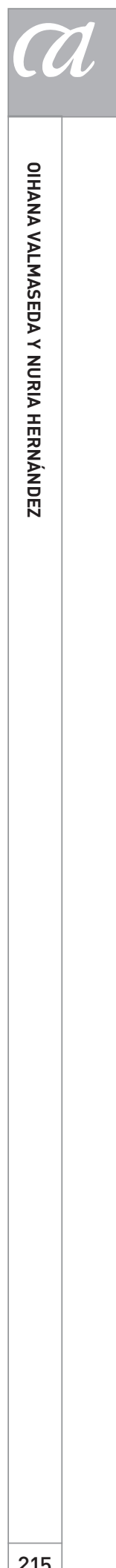


en el sistema universitario de la Comunidad Autónoma de Andalucía para las que se disponía de información en el año 2008. El registro de empresas ha sido proporcionado por la Red Andaluza de Transferencia de Resultados de la Investigación (RATRI) y la Agencia Andaluza de Innovación, siendo un total de 157 empresas las registradas hasta la fecha de referencia. En su mayoría se trata de empresas que provienen del programa de apoyo CAMPUS ${ }^{3}$. Los datos utilizados en este trabajo son resultado del desarrollo de un proyecto que, entre otros aspectos, pretendía caracterizar estructural y organizacionalmente estas empresas, estudiar su proceso de creación, así como, analizar algunos indicadores de competitividad de las mismas.

Para obtener la información necesaria se realizó una encuesta mediante entrevista personal, a través de un cuestionario dirigido a los gerentes, directivos u otra persona que hubiera tomado parte en el proceso de creación de la empresa y que conociera los elementos más relevantes de su evolución. Dado el tamaño del universo poblacional se estimó adecuado realizar la encuesta a la totalidad de la población. Finalmente, se efectuaron 121 entrevistas de las 157 posibles, obteniéndose una tasa de respuesta del 77,07\%. El cuestionario utilizado se estructuró en cinco bloques. En primer lugar, se ha obtenido información acerca de variables generales de la empresa. En el segundo bloque, se analiza todo el proceso de creación de la spinoff, desde las ideas que dieron origen a cada uno de los proyectos de empresa, motivaciones, perfil de los individuos y recursos y capacidades puestas en acción para el proyecto. Asimismo, en el tercer bloque se han examinado las relaciones que estas empresas tienen con los centros públicos de investigación y otras empresas, vinculadas con la capacidad de innovar. Igualmente, se ha explorado la importancia de la localización física de estas empresas en diferentes entornos. Finalmente, el cuarto de los bloques permite un exhaustivo análisis de la situación actual de estas empresas.

\subsection{Medición de las fuentes de conocimiento para la innovación}

Para la medición de las fuentes de conocimiento de las empresas estudiadas, se ha acudido a escalas ampliamente aceptadas y utilizadas en la literatura. Utilizando las definiciones y directrices del Manual de Oslo (OECD, 2005), se han estudiado dos esferas distintas de acceso al conoci- miento: una externa, fundamentada en las relaciones con centros públicos de investigación y otras empresas, medida a través de la variable Adquisición de $1+D$ externa. Esta variable recoge los trabajos realizados por otras organizaciones (incluidos otros grupos de empresas, organismos públicos o privados de investigación) y comprados por la empresa para aumentar el volumen de conocimientos y/o aplicación de este conocimiento en la concepción de nuevas o mejoradas aplicaciones o productos (bienes 0 servicios y/o procesos).

La dimensión interna se refiere a las actividades de I+D que la empresa realiza internamente y es medida con la variable $I+D$ interna. Esta variable recoge los trabajos realizados dentro de la empresa para aumentar el volumen de conocimientos y/o aplicación de este conocimiento en la concepción de nuevas o mejoradas aplicaciones o productos (bienes o servicios y/o procesos).

En lo que respecta a las medidas que tienen que ver con fuentes específicas de conocimiento de ámbito externo a la empresa, hemos tenido en cuenta, fundamentalmente, los trabajos de Cohen et al. (1998), Cohen et al. (2002), Perkmann y Walsh (2007) y Agrawal y Henderson (2002), en los que se identifican diferentes mecanismos a través de los cuales el tejido empresarial accede a la investigación pública. Estos trabajos exponen la amplia variedad de canales a través de los cuales es posible la obtención de conocimiento como fruto de la relación con universidades y centros públicos. De esta forma, los canales que permiten el acceso al conocimiento externo considerados en la investigación se han medido con las variables asesoramiento tecnológico, investigación contratada, realización de un proyecto de investigación conjunto, uso de instalaciones y equipos, explotación de patentes, prácticas de personal científico o técnico en la empresa, intercambio de personal, formación y las relaciones informales. Adicionalmente, hemos querido indagar, utilizando la misma escala que para el sector público de investigación, si las spin-off acceden a conocimiento que proviene de otras empresas privadas como clientes, proveedores y competidores y comprobar así si tienen un peso relevante como fuentes de conocimiento.

Por último, para comprobar si las empresas adoptan una estrategia de combinación del conocimiento que se deriva de la realización de actividades de I+D internamente y la 
adquisición externa de $I+D$, se han creado las variables $I+D$ interna y Adquisición de $I+D$ externa para los casos de las empresas que afirman emplear ambas fuentes de conocimiento en sus procesos productivos y de innovación.

\section{Resultados}

\subsection{Principales rasgos de las spin-off universitarias de Andalucía}

En línea con Pirnay et al. (2003), y adaptando su trabajo al contexto de Andalucía, se ha realizado una descripción de las empresas spin-off académicas en el sistema universitario andaluz, lo que permite dibujar el perfil de dichas empresas y comprender en mayor medida los resultados en lo referido a la combinación de actividades internas de I+D con la obtención de conocimiento de agentes externos como fuentes de conocimiento para la actividad de las empresas. En el epígrafe siguiente se estudiará la relación entre las variables descriptivas empleadas en la investigación de Pirnay et al. (2003) del perfil de spin-off y la selección de una determinada estrategia de obtención de conocimiento.
En la tabla 1 se muestran los aspectos determinantes de las empresas analizados en la literatura y la frecuencia de los mismos para los casos de spin-off observadas en la Comunidad Autónoma de Andalucía. Se representan las variables estatus de la/s persona implicada/s en la creación de la spin-off, origen de las actividades de la spin-off y la variable naturaleza del conocimiento transferido, es decir, si se trata de una empresa enfocada al diseño y desarrollo de un producto, a la prestación de un servicio o ambas.

Contrariamente a lo que concluyen algunos trabajos, en el contexto andaluz se observa gran heterogeneidad en la composición del equipo fundador de estas empresas. Por otra parte, se trata de empresas en las que destaca la explotación de un conocimiento derivado de una investigación en la universidad como principal razón que da origen a la creación de la empresa. Las spin-off analizadas se dedican tanto al diseño y desarrollo de un producto como a la prestación de servicios vinculados al mismo. Muchos trabajos recogen estas categorías de conocimiento transferido como excluyentes, circunstancia que no constatan los datos obtenidos en el contexto de esta investigación, dado el alto porcentaje de asociación entre ambas actividades.

TABLA 1. ASPECTOS DETERMINANTES DE LAS EMPRESAS SPIN-OFF

\begin{tabular}{|c|c|c|}
\hline Característica & Categoría & $\%$ \\
\hline \multirow{4}{*}{$\begin{array}{l}\text { Estatus en la universidad de los individuos } \\
\text { implicados en la creación de la empresa }\end{array}$} & Profesor & 43,77 \\
\hline & Becario pre/post & 10,63 \\
\hline & Estudiante & 13,91 \\
\hline & Personas no vinculadas a la organización de origen* & 24,33 \\
\hline \multirow{3}{*}{ Origen de las actividades de la spin-off } & Comercializar un conocimiento que procede de un proyecto de investigación & 72,8 \\
\hline & Comercializar un conocimiento desarrollado en una tesis & 24,8 \\
\hline & Explotar una patente & 29,8 \\
\hline \multirow{3}{*}{ Naturaleza del conocimiento transferido } & Diseño-desarrollo de un producto & 11,6 \\
\hline & Prestación de un servicio & 23,1 \\
\hline & Ambas & 65,3 \\
\hline
\end{tabular}

* Por organización de origen se entiende en este estudio la universidad u organismo de investigación donde nace la empresa tipo spin-off. Fuente: IESA-CSIC (E-0925). Elaboración propia. 
La literatura ha estudiado profusamente los factores estructurales y organizativos que condicionan una actuación centrada, bien en la utilización de recursos internos, en la búsqueda de conocimiento fuera de la empresa, 0 bien en la combinación de ambas estrategias. El sector de actividad, la intensidad tecnológica o la capacidad de absorción de la empresa son elementos que determinan la elección de la estrategia. Respondiendo a la pregunta de qué tipo de empresas se crean en las universidades andaluzas, en la tabla 2 se recoge la distribución porcentual de las principales características estructurales y organizativas.

TABLA 2. CARACTERISTICAS ESTRUCTURALES Y ORGANIZATIVAS DE LAS SPIN-OFF

\begin{tabular}{|c|c|c|}
\hline Variable & Categorías & $\%$ \\
\hline \multirow{2}{*}{ Año de constitución } & 1984-2004 & 33,1 \\
\hline & $2005-2008$ & 65,1 \\
\hline \multirow{11}{*}{ Organización de origen } & Universidad de Almería & 13,2 \\
\hline & Universidad de Cádiz & 17,4 \\
\hline & Universidad de Córdoba & 9,9 \\
\hline & Universidad de Granada & 15,7 \\
\hline & Universidad de Huelva & 2,5 \\
\hline & Universidad de Málaga & 19,8 \\
\hline & Universidad de Sevilla & 10,7 \\
\hline & CSIC & 3,3 \\
\hline & Hospital & 1,7 \\
\hline & Una empresa & 0,8 \\
\hline & Otros & 5,0 \\
\hline \multirow{6}{*}{$\begin{array}{l}\text { Principal área de conocimiento } \\
\text { en la que se enmarca la empresa }\end{array}$} & Ciencias Naturales (AGR+RNM) & 26,4 \\
\hline & Ciencias de la Vida (BIO + CTS) & 19,0 \\
\hline & Ciencias Exactas y Experimentales (FOM) & 5,0 \\
\hline & Humanidades (HUM) & 5,8 \\
\hline & Ciencias Sociales y Juridicas (SEJ) & 3,3 \\
\hline & Tecnologias (TEP+TIC) & 40,5 \\
\hline \multirow{9}{*}{$\begin{array}{l}\text { Actividad principal de la empresa } \\
\text { (según CNAE 09) }\end{array}$} & Agricultura, ganadería, selvicultura y pesca & 4,1 \\
\hline & Industria manufacturera & 5,0 \\
\hline & Actividades de saneamiento, gestión de residuos y contaminación & 1,7 \\
\hline & Construcción & 0,8 \\
\hline & Información y comunicaciones & 12,4 \\
\hline & Actividades profesionales, científicas y técnicas & 66,9 \\
\hline & Educación & 1,7 \\
\hline & Actividades sanitarias y de servicios sociales & 3,3 \\
\hline & Actividades artísticas, recreativas y de entretenimiento & 4,1 \\
\hline \multirow{3}{*}{ Tamaño de la empresa } & De 1 a 9 trabajadores & 73,6 \\
\hline & De 10 a 20 trabajadores & 15,7 \\
\hline & Más de 20 trabajadores & 9,9 \\
\hline \multirow{5}{*}{ Ubicación de la empresa } & Parque científico-tecnológico & 45,5 \\
\hline & Parque industrial & 9,1 \\
\hline & Zona urbana & 27,3 \\
\hline & Zona urbana-periférica & 3,3 \\
\hline & En una universidad & 12,4 \\
\hline \multirow{5}{*}{$\begin{array}{l}\text { Personal de la empresa según nivel } \\
\text { de estudios }\end{array}$} & Estudios de tercer ciclo (grado de doctor) & 18,4 \\
\hline & Estudios universitarios superiores (licenciados no incluidos anteriormente) & 45,3 \\
\hline & Estudios universitarios medios (diplomaturas) & 13,0 \\
\hline & Estudios secundarios & 18,1 \\
\hline & Estudios primarios & 4,3 \\
\hline
\end{tabular}

Fuente: IESA-CSIC (E-0925). Elaboración propia. 
En líneas generales, se trata de empresas con pocos años de actividad: el 65\% de las empresas analizadas se han constituido entre el año 2005 y 2008. Destaca como organización de origen la Universidad de Málaga, responsable de la creación de aproximadamente el $20 \%$ de las empresas, seguida de la Universidad de Cádiz $(17,4 \%)$ y de la Universidad de Granada (15,7\%). Por áreas de conocimiento, cabe resaltar el peso de las spin-off vinculadas al área de Tecnologías 4 . En segundo lugar, las Ciencias Naturales aglutinan el 26,4\% de las empresas y, a continuación, aparecen las disciplinas relacionadas con las Ciencias de la Vida, que suponen el 19\% de las spin-off de Andalucía. En comparación a estas cifras, se observa una presencia reducida de empresas en las áreas de Ciencias Exactas y Experimentales, Humanidades y Ciencias Sociales y Jurídicas, siendo estas últimas las menos presentes en la población.

En cuanto a los sectores de actividad, destacan en frecuencia las empresas dedicadas a actividades profesionales, científicas y técnicas $(66,9 \%)$. En relación al tamaño de las empresas, medido por el número de trabajadores, se comprueba que el 76,3\% de las empresas son microempresas, es decir, con menos de 10 trabajadores. En lugar de utilizar clasificaciones oficiales, con el objeto de explicar mejor la situación real de estas empresas en cuanto a su tamaño, se ha optado por recodificar la variable tamaño en tres categorias que responden de manera más precisa a la realidad observada. Estos datos, en términos de empleo se traducen en aproximadamente 900 puestos de trabajo, de los cuales el $18 \%$ corresponden a personas con grado de doctor y el $45,3 \%$ poseen estudios universitarios superiores, indicadores de la capacidad de absorción de conocimiento del personal que trabaja en estas empresas.

Los Parques Tecnológicos de la Comunidad son los que acogen fundamentalmente a estas empresas, concretamente al $45,5 \%$ de las entrevistadas. En esta región hay registrados diez parques científico-tecnológicos, entre los que destaca el Parque Tecnológico de Andalucía (PTA), situado en Málaga, y que desde su creación, hace aproximadamente 19 años, acoge al 77\% de las spin-off entrevistadas en la provincia malagueña.

\subsection{Fuentes de conocimiento para la innovación}

Los resultados obtenidos permiten vislumbrar que la adquisición de conocimiento relevante mediante la combinación de fuentes externas e internas es una estrategia que goza de gran seguimiento entre las spin-off. Concretamente, el $42,10 \%$ de empresas spin-off consultadas afirman acudir tanto a centros públicos de investigación y empresas como realizar actividades de $\mathrm{I}+\mathrm{D}$ internamente, ambas como fuentes de conocimiento y aprendizaje en el desarrollo de su actividad. Este dato confirma para este tipo de empresas la interdependencia y complementariedad de ambas vías para obtener conocimiento, en el sentido de que permite el enriquecimiento mutuo de ambas estrategias y efectos más positivos en comparación con la implementación exclusiva de cualquiera de las opciones. No obstante, la realización de actividades de I+D interna destaca como la estrategia de obtención de conocimiento más frecuente, concretamente en el 86,6\% de los casos estudiados, seguida por la adquisición de I+D externa en el 45,5\%.

Las características de estas empresas, tales como la elevada cualificación de su personal y el desempeño de actividades muy relacionadas con la investigación, encaminadas al desarrollo de productos y servicios de alto valor añadido, explican un comportamiento muy activo de vigilancia del entorno. También explican la existencia de las capacidades necesarias para absorber, asimilar y aplicar el conocimiento externo. El 76\% afirma realizar tareas de vigilancia sistemática del mercado $y$, además, el $48 \%$ cuenta con mecanismos para conocer las necesidades y el nivel de satisfacción de los clientes. Esto muestra el interés de estas empresas por obtener información de estas fuentes, gestionarla y utilizarla convenientemente en el desarrollo de su actividad. Estos datos, a su vez, entroncan con una atribución de importancia muy alta a las relaciones de colaboración con agentes externos. Así, tal y como se recoge en el gráfico 1, las relaciones con otros agentes tienen un peso muy importante incluso para la creación de la propia spin-off. El 77,7\% consideró que las relaciones previas a la creación de la empresa son un factor importante que contribuyó a la creación de la misma. Asimismo, el 85,1\% afirman que la interacción con centros públicos de investigación $u$ otras empresas mejora la productividad y los resultados de la empresa en I+D. La actitud de estas empresas se completa con perspectivas de futuro muy favorables con respecto a la colaboración con agentes, pues el 74\% auguraron para los próximos ejercicios un aumento de relaciones con el entorno.

ARBOR Vol. 188753 enero-febrero [2012] 211-228 ISSN: 0210-1963 
GRÁFICO 1. INDIQUE SU GRADO DE ACUERDO O DESACUERDO CON LAS SIGUIENTES AFIRMACIONES RESPECTO A LA COOPERACIÓN DE LA EMPRESA CON OTROS ORGANISMOS: LAS RELACIONES DE COLABORACIÓN...

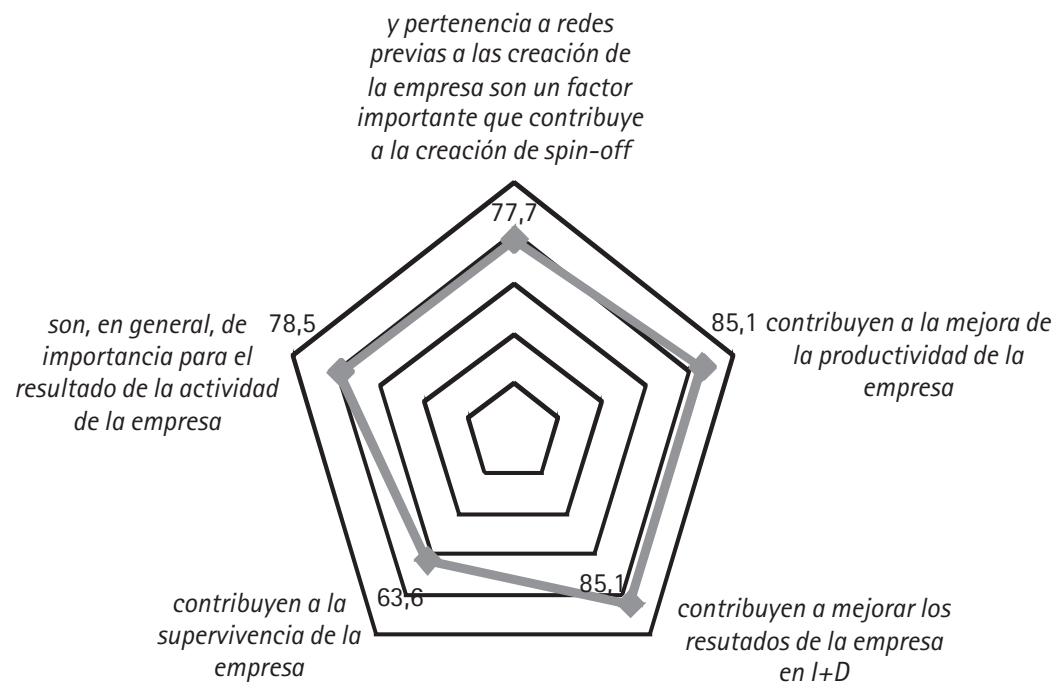

Fuente: IESA-CSIC (E-0925). Elaboración propia.

Todo ello se traduce en que el 45,5\% de las spin-off de Andalucia afirman realizar actividades vinculadas con la adquisición de I+D externa. Para profundizar más en esta cuestión, en el gráfico 2 se expone la variedad de canales a través de los cuales las spin-off acceden al conocimiento que requieren y el agente que eligen como proveedor de dicho conocimiento, distinguiendo entre otras empresas y universidades y/o OPIS.

Inicialmente, una primera lectura de este gráfico apunta a que las spin-off otorgan un papel más relevante a los centros públicos de investigación como fuentes de conocimiento. Si observamos los diferentes canales de interacción que se establecen entre las spin-off y los agentes considerados, en todos resulta ser la investigación pública la fuente principal. En el caso del asesoramiento tecnológico y consultoría y en las actividades vinculadas al alquiler de instalaciones, se encuentra menor diferencia a favor de los centros públicos de investigación. El 41,3\% de las empresas indican haber solicitado un servicio de asesoramiento tecnológico a universidades y/o OPI y un $36,4 \%$ a empresas. En el caso del alquiler de instalaciones, las spin-off han preferido a otras empresas en un 22,3\% de los casos y a una universidad y/o OPI en un $24 \%$.
Se encuentran diferencias significativas a favor de los centros públicos de investigación en las actividades relacionadas con la formación especializada, el intercambio de personal científico y la realización de prácticas. Así, entre aquellas empresas spin-off que afirman haber recibido personal especializado para la realización de prácticas, en el $69,4 \%$ de los casos resulta ser personal que proviene de universidades y OPIs. Por tanto, en todos los canales de interacción en los que el conocimiento intercambiado está basado en acciones de movilidad y formación de personal, las universidades y OPIs, como organizaciones origen de esos recursos humanos, son una fuente de conocimiento indiscutible para las empresas spin-off en el contexto andaluz. Este mismo comportamiento se aprecia en lo que respecta a la formación específica, demandada en mayor medida a los centros públicos de investigación, concretamente, en un $43,8 \%$ de los casos frente a un $33,1 \%$ de las spin-off que indican recibir dicha formación de otras empresas.

Los datos de este trabajo también confirman la importancia que este tipo de empresas otorgan a la realización de proyectos de investigación, tanto conjuntos como contratados. Son importantes vías de obtención de conocimiento 
GRÁFICO 2. DISTRIBUCIÓN PORCENTUAL DE LAS FUENTES DE CONOCIMIENTO EXTERNAS UTILIZADAS POR LAS SPIN-OFFS

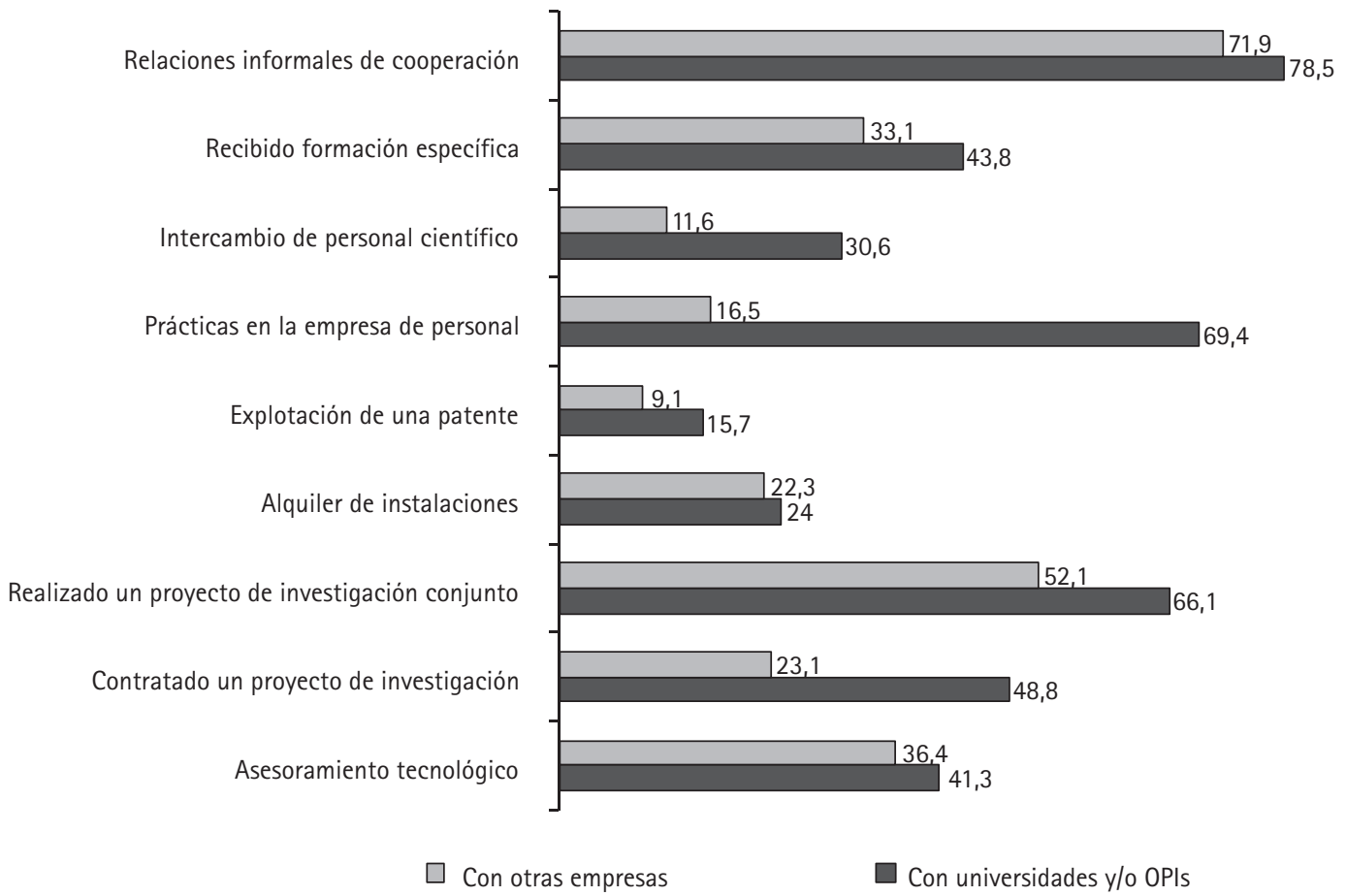

Fuente: IESA-CSIC (E-0925). Elaboración propia. Resumen de los resultados de las respuestas afirmativas.

en las que, de nuevo, los centros públicos de investigación adquieren mayor protagonismo como proveedores de conocimiento, concretamente, en un $66,1 \%$ de los casos analizados. No obstante, cabe destacar que el $52,1 \%$ de las spin-off consultadas afirman haber realizado un proyecto de investigación conjunto con otras empresas.

En los extremos destacan, de un lado, los altos porcentajes en las relaciones informales con ambos agentes $y_{\text {, de }}$ otro, la baja realización de actividades relacionadas con la explotación de patentes tanto con el sector público como con el privado, resultado este último que confirma las tesis establecidas en la literatura de que las patentes sólo son utilizadas como canal de obtención de conocimiento y de aprendizaje por un grupo especifico de empresas.

A su vez, este comportamiento atento al conocimiento que generan otros agentes externos enriquece de manera destacada la realización de actividades de I+D en el ámbito interno, como confirman el $42,1 \%$ de las empresas que indican desarrollar una estrategia abierta de obtención de conocimiento. Este tipo de empresas, dadas sus caracteristicas específicas consideran necesario tanto el conocimiento que se genera internamente como el que obtienen de organizaciones externas.

En una segunda fase de análisis de los datos se ha estudiado la asociación entre determinadas características de las empresas y la selección de una determinada estrategia de adquisición de conocimiento. En primer lugar, en la tabla 3 se recoge la relación entre las variables empleadas para la descripción de las empresas académicas del sistema universitario andaluz, es decir, el estatus de las personas implicadas en la creación de la empresa, el origen de la creación de la empresa y la naturaleza del conocimiento transferido (véase tabla 1), y la estrategia escogida para obtener el conocimiento requerido por la actividad de la empresa.

ARBOR Vol. 188753 enero-febrero [2012] 211-228 ISSN: 0210-1963 
TABLA 3. DISTRIBUCIÓN PORCENTUAL DE LA REALIZACIÓN DE ACTIVIDADES DE I+D INTERNA, EXTERNA Y AMBAS A PARTIR DE LAS VARIABLES DE LA TIPOLOGÍA DE EMPRESAS OBSERVADA

\begin{tabular}{|c|c|c|c|c|}
\hline & & I+D Interna & $\begin{array}{l}\text { Adquisición } \\
\text { de I+D externa }\end{array}$ & $\begin{array}{l}\text { I+D Interna y } \\
\text { adquisición } \\
\text { de I+D externa }\end{array}$ \\
\hline \multirow{4}{*}{$\begin{array}{l}\text { Estatus en la universidad de } \\
\text { los individuos implicados en } \\
\text { la creación de la empresa* }\end{array}$} & Profesor & 2,67 & 3,71 & 3,76 \\
\hline & Becario pre/post & 0,48 & 0,45 & 0,49 \\
\hline & Estudiante & 1,34 & 2,00 & 2,16 \\
\hline & Personas no vinculadas a la organización de origen[1] & 0,92 & 1,13 & 1,08 \\
\hline \multirow{3}{*}{$\begin{array}{l}\text { Origen de las actividades } \\
\text { de la spin-off }\end{array}$} & $\begin{array}{l}\text { Comercializar un conocimiento que procede de un proyecto } \\
\text { de investigación }\end{array}$ & $87,90 \%$ & $49,50 \%$ & $45,10 \%$ \\
\hline & $\begin{array}{l}\text { Comercializar un conocimiento desarrollado en una tesis } \\
\text { doctoral }\end{array}$ & $90,00 \%$ & $53,30 \%$ & $50,00 \%$ \\
\hline & Explotar una patente & $91,70 \%$ & $47,20 \%$ & $44,40 \%$ \\
\hline \multirow{3}{*}{$\begin{array}{l}\text { Naturaleza del conocimiento } \\
\text { transferido }\end{array}$} & Diseño-desarrollo de un producto & $78,60 \%$ & $42,90 \%$ & $35,70 \%$ \\
\hline & Prestación de un servicio & $71,40 \%$ & $53,60 \%$ & $50,00 \%$ \\
\hline & Ambas & $93,70 \%$ & $43,00 \%$ & $40,50 \%$ \\
\hline
\end{tabular}

[1] \% de las empresas que realizan las actividades indicadas en cada una de las categorías de empresa.

* Resultados medios sobre el total de spin-off.

** Identificados como "importantes" o "muy importantes" para la creación de la spin-off.

Los datos no permiten detectar diferencias importantes en las estrategias atendiendo a la composición del grupo fundador de las empresas, si bien sí es posible apreciar que la realización de I+D externa y la implementación de una estrategia combinada se asocian a grupos de individuos sensiblemente mayores, que superan los valores medios observados, aunque sin diferencias en cuanto al perfil.

La explotación de una patente y la comercialización de un conocimiento desarrollado en una tesis doctoral se encuentra muy relacionada con la realización de $\mathrm{I}+\mathrm{D}$ interna. Casi el 92\% de las empresas que afirman haber explotado una patente y el $90 \%$ de las empresas creadas para la comercialización del conocimiento derivado de una tesis doctoral realizan I+D interna. Sin embargo, las razones que con mayor frecuencia e importancia desencadenaron la creación de las spin-off no discrimina en gran medida en lo que a realización de actividades de I+D interna se refiere. Donde más diferencia porcentual se detecta es en la asociación entre la comercialización de un cono- cimiento resultante de una tesis doctoral y la consulta tanto de fuentes internas como externas por parte de las empresas.

En cuanto al tipo de conocimiento transferido, las empresas que se dedican al diseño y fabricación de un producto así como a la prestación de servicios relacionados con dicho producto presentan valores muy altos en la realización de I+D interna. Por otra parte, se identifica una relación importante entre la prestación de servicios y la realización de I+D externa. El 53,6\% de las empresas cuya actividad se basa en la prestación de servicios indican adquirir I+D externa. Asimismo, se trata de este tipo de empresas las que con mayor intensidad optan por combinar tanto conocimiento derivado de las actividades de I+D interna como externa.

En segundo lugar, en la tabla 4 se estudia la relación entre variables estructurales como el área de conocimiento de la empresa, el sector de actividad, el tamaño empresarial 
TABLA 4. DISTRIBUCIÓN PORCENTUAL DE LA REALIZACIÓN DE ACTIVIDADES DE I+D INTERNA, EXTERNA Y AMBAS SEGÚN VARIABLES ESTRUCTURALES

\begin{tabular}{|c|c|c|c|c|}
\hline & $\begin{array}{l}\text { I+D Interna } \\
\quad(\%)\end{array}$ & $\begin{array}{l}\text { Adquisición de I+D } \\
\text { externa (\%) }\end{array}$ & $\begin{array}{c}\text { I+D Interna y } \\
\text { adquisición de I+D } \\
\text { externa (\%) }\end{array}$ & Total \\
\hline$\%$ total de empresas que realizan estas actividades & 86,8 & 45,5 & 42,1 & \\
\hline Área de conocimiento & $* * *$ & & & \\
\hline Ciencias Naturales (AGR+RNM) & $87,50 \%$ & $43,80 \%$ & $40,60 \%$ & $100,00 \%$ \\
\hline Ciencias de la Vida (BIO + CTS) & $87,00 \%$ & $69,60 \%$ & $60,90 \%$ & $100,00 \%$ \\
\hline Ciencias Exactas y Experimentales (FOM) & $100,00 \%$ & $33,30 \%$ & $33,30 \%$ & $100,00 \%$ \\
\hline Humanidades & $57,10 \%$ & $14,30 \%$ & $14,30 \%$ & $100,00 \%$ \\
\hline Ciencias Sociales y Juridicas & $25,00 \%$ & $50,00 \%$ & $25,00 \%$ & $100,00 \%$ \\
\hline Tecnologias (TEP+TIC) & $93,90 \%$ & $40,80 \%$ & $40,80 \%$ & $100,00 \%$ \\
\hline Sector de Actividad (CNAE09) & *** & & & \\
\hline Agricultura, ganadería, selvicultura y pesca & $40,00 \%$ & $60,00 \%$ & $20,00 \%$ & $100,00 \%$ \\
\hline Industria manufacturera & $100,00 \%$ & $50,00 \%$ & $50,00 \%$ & $100,00 \%$ \\
\hline $\begin{array}{l}\text { Actividades de saneamiento, gestión de residuos y con- } \\
\text { taminación }\end{array}$ & $100,00 \%$ & $50,00 \%$ & $50,00 \%$ & $100,00 \%$ \\
\hline Construcción & $100,00 \%$ & & & $100,00 \%$ \\
\hline Información y comunicaciones & $100,00 \%$ & $46,70 \%$ & $46,70 \%$ & $100,00 \%$ \\
\hline Actividades profesionales, científicas y técnicas & $90,10 \%$ & $46,90 \%$ & $45,70 \%$ & $100,00 \%$ \\
\hline Educación & $50,00 \%$ & $50,00 \%$ & & $100,00 \%$ \\
\hline Actividades sanitarias y de servicios sociales & $50,00 \%$ & $25,00 \%$ & $25,00 \%$ & $100,00 \%$ \\
\hline Actividades artísticas, recreativas y de entretenimiento & $60,00 \%$ & $20,00 \%$ & $20,00 \%$ & $100,00 \%$ \\
\hline N. ${ }^{\circ}$ de trabajadores & & $* * *$ & *** & \\
\hline De 1 a 9 trabajadores & $84,30 \%$ & $37,10 \%$ & $33,70 \%$ & $100,00 \%$ \\
\hline De 10 a 20 trabajadores & $94,70 \%$ & $57,90 \%$ & $52,60 \%$ & $100,00 \%$ \\
\hline Más de 20 trabajadores & $100,00 \%$ & $91,70 \%$ & $91,70 \%$ & $100,00 \%$ \\
\hline Con clientes en la UE & $88,50 \%$ & $57,70 \%$ & $51,90 \%$ & $100,00 \%$ \\
\hline Con clientes en otros paises fuera de la UE & $84,80 \%$ & $58,70 \%$ & $52,20 \%$ & $100,00 \%$ \\
\hline
\end{tabular}

Representados solamente los porcentajes de "SI".

$* * *$ Chi-cuadrado $\leq 0,005$.

Fuente: IESA-CSIC (E-0925). Elaboración propia.

y la realización de actividad exportadora, ampliamente utilizadas en investigaciones previas como factores determinantes en la elección de las fuentes de conocimiento.
Por área de conocimiento, se advierte una importante asociación entre las áreas de conocimiento Ciencias Exactas y Experimentales y Tecnologías y la realización de I+D

ARBOR Vol. 188753 enero-febrero [2012] 211-228 ISSN: 0210-1963 
interna. Sin embargo, son las áreas de Ciencias de la Vida y Ciencias Sociales las que más se relacionan con la adquisición de I+D externa. Por último, y con una diferencia destacada, las empresas de Ciencias de la Vida son las que más se asocian con la estrategia combinada de I+D interna y externa. Concretamente, el 61\% de las empresas de ese campo científico implementan ambas opciones simultáneamente.

Los sectores de actividad que claramente se vinculan con las actividades de I+D interna como fuente de conocimiento son la industria manufacturera, el sector de actividades de saneamiento, gestión de residuos y contaminación, las empresas de construcción y, por último, el sector de información y comunicaciones. El 100\% de las empresas de estos sectores afirman que las actividades de I+D internas son una importante fuente de conocimiento. De igual forma lo son para el $90 \%$ de las empresas dedicadas a actividades profesionales, científicas y técnicas.

La utilización de manera combinada de actividades de I+D internas y externas está presente en mayor medida entre las empresas manufactureras y las dedicadas a actividades de saneamiento, gestión de residuos y contaminación.

Con respecto al tamaño de las empresas medido por el número de trabajadores, los datos permiten observar la influencia de esta variable en el tipo de fuentes de conocimiento a las que se accede. Lo que queda patente es que a mayor número de trabajadores mayor es el porcentaje de empresas que realizan I+D interna, externa o la combinación de ambas. Se trata de una variable importante que marca una tendencia clara en lo que a selección de fuentes de conocimiento se refiere. También se ha confirmado una asociación importante entre la internacionalización de la actividad empresarial y la combinación de fuentes de conocimiento.

\section{Conclusiones}

La literatura relativa al estudio de los procesos de innovación empresarial ha evolucionado desde posiciones que consideraban la innovación como un proceso lineal hasta planteamientos que, asumiendo la complejidad del proceso innovador, defienden la necesidad de acceder a diversas fuentes de conocimiento, tanto a las que tienen como origen las actividades internas de la organización como a las que, por el contrario, provienen de agentes externos a la empresa. Introducir nuevos productos en el mercado o mejorar los existentes con éxito plantea a la empresa una necesidad de apertura a fuentes de conocimiento de diversos actores que, inevitablemente, requiere al mismo tiempo la habilidad de reconocer y poner en valor el conocimiento disponible. Esta habilidad se resume en el concepto de capacidad de absorción (Cohen y Levinthal, 1990) y determina la definición de una estrategia de búsqueda de conocimiento adecuada en las empresas.

En línea con investigaciones que apuntan la conveniencia de contemplar las fuentes de conocimiento internas y externas como complementarias (Mowery, 1983; Cassiman y Veugelers, 2006; Romijn et al., 2002; Freel 2003; Freel 2005; Caloghirou et al., 2004), los análisis realizados revelan la combinación de ambas estrategias de adquisición de conocimiento en las spin-off del contexto académico andaluz, constituyendo un recurso muy frecuente en las empresas consultadas. El desarrollo conjunto de actividades de $I+D$ internas y procesos de búsqueda de conocimiento externo tiene lugar en el $42 \%$ de las spin-off analizadas. Aunque conviene señalar que la fuente principal de conocimiento se halla en la propia actividad interna de I+D tal y como indican aproximadamente el $87 \%$ de las empresas. Asimismo, se trata de empresas muy activas en la búsqueda de conocimiento de agentes como universidades, OPIs y otras empresas privadas, comportamiento desarrollado por el $45,5 \%$ de las empresas.

El estudio también ha permitido identificar las variables que más se asocian con una estrategia de adquisición de conocimiento concreta. Se ha comprobado una relación importante entre determinadas áreas de conocimiento y la selección de una determinada fuente de conocimiento. Así, se conoce que el 100\% de las empresas de ciencias exactas y experimentales y el $94 \%$ de Tecnologías realizan I+D interna. Sin embargo, en cuanto a la realización de I+D externa y a la combinación de I+D interna y externa la asociación es más fuerte con las empresas de Ciencias de la Vida.

Otra caracteristica empresarial habitualmente estudiada como factor determinante de la estrategia de innovación de las empresas es el tamaño empresarial. En este sentido, 
encontramos que a mayor número de trabajadores en la empresa también es mayor la intensidad en la asociación de esta variable con cualquiera de las fuentes de conocimiento consideradas. Un aspecto que también discrimina el comportamiento de las empresas es si tienen mercado internacional. Así, se observa que las empresas internacionalizadas destacan tanto en la realización de I+D interna, externa como ambas.

Profundizando más en las relaciones que estas empresas mantienen con agentes como universidades y OPIs y otras empresas, es posible observar un papel destacado de los primeros como proveedores de conocimiento relevante. Las universidades y OPIs son los agentes por excelencia a los que acceden las spin-off para obtener formación específica y de alta cualificación, para acoger en sus instalaciones personal en prácticas y para realizar intercambios de personal científico. Este hecho puede estar condicionado, tal y como señala la perspectiva institucional, por la influencia de la organización de origen tanto en la configuración de la propia empresa como en su posterior desarrollo (Carayannis et al., 1998; Steffensen et al., 2000; Meyer, 2003; Moray y Clarysse, 2005; Clarysse et al., 2005). Por el contrario, los resultados muestran una posición más igualada entre agentes públicos y privados en lo que a alquiler de instalaciones y maquinaria se refiere, así como en el acceso a asesoramiento tecnológico y consultoría.

El estudio permite concluir que el origen académico de estas empresas influye en el hecho de que accedan frecuentemente a conocimiento de fuentes públicas y que prevean un fortalecimiento de las relaciones con la universidad como pilares de su estrategia de innovación. Al mismo tiempo, este tipo de empresas encuentran en la realización de actividades internas de I+D las capacidades necesarias para el aprovechamiento adecuado de las fuentes externas. Por tanto, el propio origen de la empresa está muy asociado al interés que estas empresas manifiestan tanto en la realización de actividades de I+D como en su adquisición externa.

Este trabajo constituye una aproximación al estudio de un fenómeno que ha sido abordado en estudios previos, aunque escasamente para el caso de empresas con origen académico. Por ello, las conclusiones obtenidas suponen un avance en la descripción de las fuentes de conocimiento utilizadas por esta tipología de empresa así como en la identificación de las características que más influyen en la elección de una estrategia concreta de adquisición del conocimiento que resulta necesario incorporar en los procesos productivos y de innovación. El estudio ofrece implicaciones importantes para las políticas relacionadas con el apoyo a la creación de empresas. En primer lugar, destaca que la mayor parte de las empresas que surgen de las universidades no emplean conocimiento codificado en patentes, sino una variedad de capacidades generadas por la investigación pública. En segundo lugar, resalta que las empresas existentes se caracterizan por complementar los conocimientos procedentes de la universidad con otra serie de fuentes muy variadas. Las claves de la competitividad de estas empresas posiblemente tienen que ver con su estrategia de innovación abierta, que las hace buscar conomiento en aquellos lugares en los que está disponible, y no las hace dependientes de la organización de la que surgen. En tercer lugar, destaca el importante papel del sector público como proveedor de recursos humanos y formación, que constituyen las claves del desarrollo de este tipo de empresas.

\section{NOTAS}

1 Las autoras agradecen a Teresa González de la Fe y a Manuel Fernández Esquinas su importante contribución en el artículo.

2 Estudio E-0925 IESA-CSIC. "Creación de empresas y actividades de transfe-

Recibido: 10 de abril de 2011

Aceptado: 23 de julio de 2011 rencia de conocimiento en el sistema público de I+D de Andalucía". Pro- yecto financiado por la Consejería de Innovación, Ciencia y Empresa de la Junta de Andalucía

3 La Consejería de Innovación, Ciencia y Empresa, a través de la Agencia de Innovación y Desarrollo de Andalucía y las universidades andaluzas, ha impulsado el denominado Proyecto Campus. Se trata de un programa de incentivos económicos que consiste, 
fundamentalmente, en el impulso de los recursos propios de las empresas de base tecnológica de nueva creación.

$4 \mathrm{El}$ área de Tecnologías incluye las Tecnologías de la Producción y Construcción y las Tecnologías de la Información y Comunicación. Ciencias Naturales incluye las disciplinas de Agroalimentación y Recursos Naturales y Medio Ambiente.

\section{BIBLIOGRAFÍA}

Acs, Z. J.; Audretsch, D. B. y Feldman, M. P. (1994): "R\&D spillovers and recipient firm size", Review of Economics and Statistics, 76, 336-340.

Agrawal, A. y Henderson, R. (2002): "Putting Patents in context: Exploring Knowledge Transfer from MIT", $M a-$ nagement Science, 48, 44-60.

Autio, E. (1997): "New technology-based firms in innovation networks", Research Policy, 26, 263-281.

Autio, E. y Lumme, A. (1998): "Does the innovator role affect the perceived potencial for growth? Analysis of four types of new technology-based firms", Technology Analysis \& Strategic Management, 10, 41-54.

Barney, J. (1991): "Firm resources and sustained competitive advantage", Journal of Management, 17, 99-120.

Barney, J.; Wright, M. y Ketchen, D. (2001): "The resource based view: 10 years after", Journal of Management, 27, 625-642.

Bellini, E.; Capaldo, G.; Edström, A.; Kaulio, M.; Raffa, M.; Riccardia, M. y Zollo, G. (1999): "Strategic paths of academic spin-offs: A Comparative Analysis of Italian and Swedish Cases", 44th ICSB Conference, Naples, 20-23 June.

Bender, G. y Laestadius, S. (2005): "Non science based innovativeness: on ca- pabilities relevant to generate profitable novelty", en Bender, G., Jacobson, D., y Robertson, P. L. (eds.), "Non-Research- Intensive Industries in the Knowledge Economy", Journal for Perspectives on Economic Political and Social Integration, 11, 123-170.

Bower, D. (2003): "Business model fashion and the academic spinout firm", $R \& D$ Management, 33, 97-105.

Brush, C. G.; Greene, P. G. y Hart, M. M. (2001): "From initial idea to unique advantage: The entrepreneurial challenge of constructing a resource base", Academy of Management Executive, 15, 64-78.

Caloghirou, Y.; Kastelli, I. y Tsakanikas, A. (2004): "Internal capabilities and external knowledge sources: complements or substitutes for innovative performance?", Technovation, 24, 2939.

Carayannis, E.; Rogers, E.; Kurihara, K. y Allbritton, M. (1998): "High technology spin-offs from government R\&D laboratories and research institutes", Technovation, 18, 1-10.

Cassiman, B. y Veugelers, R. (2006): "In search of complementarity in innovation strategy: internal R\&D and external knowledge acquisition", $M a-$ nagement Science, 52, 68-82.

Chesbrough, H. y Rosenbloom, R. S. (2002): "The role of business model in capturing value from innovation", Industrial and Corporate Change, 11, 529-544.

Chiesa, V. y Piccaluga, A. (2000): "Exploitation and diffusion of public research: the general framework and the case of academic spin-off companies", R\&D Management, 30, 329-340.

Clarysse, B.; Heirman, A. y Degroof, J. (2000): "An Institutional and Resource Based Explanation of Growth Patterns of Research based Spin-Offs in Europe", Frontiers of Entrepreneurial Studies, 545-559.
Clarysse, B.; Wright, M.; Lockett, A.; van de Velde, E. y Vohora, A. (2005): "Spinning out new ventures: a typology of incubation strategies from European research institutions", Journal of Business Venturing, 20, 183-216.

Cohen, W. M. y Levinthal, D. (1989): "Innovation and Learning: the two faces of R\&D", The Economic Journal, 99, 569-596.

Cohen, W. M. y Levinthal, D. (1990): "Absorptive-capacity-a new perspective on learning and innovation", Administrative Science Quarterly, 35, 128152.

Cohen, W. M.; Florida, R.; Randazzese, L. y Walsh, J. (1998): "Industry and the academy: Uneasy partners in the cause of technological advance", en R. Noll (eds.), Challenges to Research Universities, Brookings Institution Press, Washington, D.C.

Cohen, W. M.; Nelson, R. y Walsh, J. (2002): "Links and Impacts: The influence of public research on industrial R\&D", Management Science, 48, 1-23.

Druilhe, C. y Garnsey, E. (2004): "Do academic spin-outs differ and does it matter?", Journal of Technology Transfer, 29, 269-285.

Etzkowitz, H. y Leydesdorff, L. (1997): Universities in the Global Knowledge Economy: A Triple Helix of AcademicIndustry-Government Relations, London, Cassell.

Fontes, M. (2001): "Biotechnology entrepreneurs and technology transfer in a intermediate economy", Technological Forecasting and Social Change, $62,59-74$.

Freel, M. (2003): "Sectoral patterns of small firm innovation, networking and proximity", Research Policy, 32, 751-770.

Freel, M. (2005): "Patterns of innovation and skills in small firms", Technovation, 25, 123-134. 
Grimpe, C. y Sofka, W. (2009): "Search patterns and absorptive capacity: Low- and high-technology sectors in European countries", Research Policy, 38, 495-506.

Gibbons, M.; Limoges, C.; Nowotny, H.; Schwartzman, S.; Scott, P. y Trow, M. (1994): The New Production of Knowledge: The Dynamics of Science and Research in Contemporary Societies, London, Sage Publications.

Heirman, A. y Clarysse, B. (2004): "How and why do research-based start-ups differ at founding? A resource-based configurational perspective", Journal of Technology Transfer, 29, 247-268.

Hellmann, T. y Puri, M. (2000): "The interaction between product market and financing strategy: the role of venture capital", Review of Financial Studies, 13, 959-984.

Hindle, K. y Yencken, J. (2004): "Public research commercialisation, entrepreneurship and new technology based firms: an integrated model", Technovation, 24, 793-803.

Katila, R. (2002): "New product search over time: past ideas in their prime?" Academy of Management Journal, 45, 995-1010.

Katila, R. y Ahuja, G. (2002): "Something old, something new: a longitudinal study of search behaviour and new product introduction", Academy of Management Journal, 45, 11831194.

Kline, S. y Rosenberg, N. (1986): "An overview of innovation", en Landau, R. y Rosenberg, N. (eds.), The Positive Sum Strategy: Harnessing Technology for Economic Growth, Washington, National Academic Press.

Klofsten, M. y Jones-Evans, D. (2000): "Comparing Academic Entrepreneurship in Europe -The Case of Sweden and Ireland", Small Business Economics, 14, 299-309.
Lane, P. J.; Koka, B. R. y Pathak, S. (2006): "The reification of absorptive capacity: a critical review and rejuvenation of the construct", Academy of Management Review, 31, 833-863.

Laursen, K. y Salter, A. (2006): "Open for Innovation: The role of openness in explaining innovative performance among U.K. manufacturing firms", Strategic Management Journal, 27, 131-150.

Lindelhof, P. y Lofsten, H. (2004): "Proximity as a resource base for competitive advantage: university-industry links for technology transfer", Journal of Technology Transfer, 29, 311-326.

Link, A. L. y Rees, J. (1990): "Firm size, university based research, and the returns to R\&D", Small Business Economics, 2, 25-31.

March, J. C. (1991): "Exploration and exploitation in organization learning", Organization Science, 2, 71-87.

Meyer, M. (2003): "Academic entrepreneurs or entrepreneurial academics? Research-based ventures and public support mechanisms", R\&D Management, 33, 107-115.

Moray, N. y Clarysse, B. (2005): "Institutional change and resource endowments to science-based entrepreneurial firms", Research Policy, 34, 1010-1027.

Mowery, D. C. (1983): "The relationship between intrafirm and contractual forms of industrial research in American manufacturing, 1900-1940", Exploration in Economics History, 20, 351-374.

Mustar, P. (1997): "Spin-off enterprises: how French academies create hi-tech companies: the condition for success or failure", Science and Public Policy, 24, 37-43.

Mustar, P. (2000): "New business venturing by researchers", Bordeaux, 9-10 March.

Mustar, P. (2002): "Public support for the spin-off companies from higher educations and research institution",
Conference STRATA Brussels, 22-23 April 2002.

Mustar, P.; Renault, M.; Colombo, M.; Piva, E.; Fontes, M.; Lockett, A.; Wright, M.; Clarysse, B. y Moray, N. (2006): "Conceptualising the heterogeneity of research-based spin-offs: A multidimensional taxonomy", Research Policy, 35, 289-308.

Nicolaou, N. y Birley, S. (2003): "Academic networks in a trichotomous categorisation of university spinouts", Journal of Business Venturing, 18, 333-359.

OCDE (2005): Manual de Oslo. Directrices para la recogida e interpretación de datos sobre innovación.

OCDE (1997): Révision des classifications des secteurs et des produits de haute technologie. Doc. OCDE/GD (97)216.

Perkmann, M. y Walsh, K. (2007): "University-industry relationships and open innovation: towards a research agenda", International Journal of Management Reviews, 9, 259-280.

Pirnay, F. (1998): "What are we talking about when we talk about spin-off? A review of the literature", 4ème CoIloque International Francophone sur la PME, Metz Nancy, 22-24 October.

Pirnay, F.; Surlemont, B. y Nlemvo, F. (2003): "Toward a Tipology of University Spinoff", Small Business Economics, 21, 355-369.

Rappert, B.; Webster, A. y Charles, D. (1999): "Making Sense of Diversity and Reluctance: Academic-Industrial Relations and Intellectual Property", Research Policy, 28, 873-890.

Romijn, H. y Albaladejo, M. (2002): "Determinants of innovation capability in small electronics and software firms in southeast England", Research Policy, 31, 1053-1067.

Santamaría, L.; Nieto, M. J. y Barge-Gil, A. (2009): "Beyond formal R\&D: Taking advantage of other sources of innovation in low- and medium- technology 
industries", Research Policy, 38, 507517.

Scholten, V.; Omta, 0. y Elfring, T. (2001): "The significance of partenthood: the social capital of the parent organization and the entrepreneurial outcome". Rent XV Conference, Finland.

Shan, W.; Walker, G. y Kogut, B. (1994): "Interfirm cooperation and start-up innovation in the biotechnology industry", Strategic Management Journal, 22, 387-394.

Shane, S. y Stuart, T. (2002): "Organizational endowments and the performance of university start-ups", Management Science, 48, 154-170.
Smirlor, R.; Gibson, D. y Dietrich, B. (1990): "Spin-Out Companies: Technology Start-ups from UT-Austin", Journal of Business Venturing, 5, 63-76.

Stankiewicz, R. (1994): "University firms: Spin-off companies from universities", Science and Public Policy, 21, 99-107.

Steffensen, M.; Rogers, E. M. y Speakman, K. (2000): "Spin-offs from research centers at a research university", Journal of Business Venturing, 15, 93111.

Tidd, J. y Trewhella, M. (1997): "Organizational and technological antecedents for knowledge acquisition and lear- ning", R\&D Management, 24, 359375.

Tidd, J. (2000): From Knowledge Management to Strategic Competence: Measuring Technological, Market and Organisational Innovation, London, Imperian College Press.

Wright, M.; Vohor, A. y Lockett, A. (2004): "Entrepreneurship and university technology transfer", Journal of Technology Transfer, 29, 235-246.

Zott, C. y Amit, R. (2005): "Business Model" en Hitt, M. y Ireland, D. (eds.), The Blackwell Encyclopedia of Management. Entrepreneurship, 3, 20-24, Oxford, Blackwell Publishing. 